\title{
A Common Framework for Artificial Intelligence in Higher Education (AAI-HE Model)
}

\author{
Thiti Jantakun ${ }^{1}$, Kitsadaporn Jantakun ${ }^{1} \&$ Thada Jantakoon ${ }^{2}$ \\ ${ }^{1}$ Department of Computer Education, Faculty of Education, Roi Et Rajabhat University, Roi Et, Thailand \\ ${ }^{2}$ Department of Information and Communication Technology for Education, Faculty of Science and Technology, \\ Rajabhat Maha Sarakham University, Maha Sarakham, Thailand \\ Correspondence: Thada Jantakoon, Department of Information and Communication Technology for Education, \\ Faculty of Science and Technology, Rajabhat Maha Sarakham University, Maha Sarakham, Thailand.
}

Received: April 14, 2021

Accepted: August 2, $2021 \quad$ Online Published: October 28, 2021

doi:10.5539/ies.v14n11p94

URL: https://doi.org/10.5539/ies.v14n11p94

\begin{abstract}
This research aims to 1) Develop a common framework for artificial intelligence in higher education (AAI-HE model) and 2) Assess the AAI-HE model. The research process is divided into two stages: 1) Develop an AAI-HE model, and 2) Assessment the model. The sample consists of five experts chosen through purposive sampling. The data is analyzed by means and standardized deviations statistically. The research result shows that 1) the AAI-HE model consists of seven key components which are 1.1) User Interactive Components and Technology of AI, 1.2) Components and Technology of AI, 1.3) Roles for Artificial Intelligence in Education 1.4) Machine Learning and Deep Learning 1.5) DSS Modules 1.6) Applications of Artificial Intelligence in Education, and 1.7) AI to enhance campus efficiencies, and 2) The result of the assessment of the AAI-HE model is rated as absolutely appropriate overall.
\end{abstract}

Keywords: artificial intelligence, artificial intelligence in higher education, AAI-HE model

\section{Introduction}

$\mathrm{AI}$ is a concept developed by John McCarthy in 1955 to represent a computer that can execute a range of human cognitive skills such as speaking, thinking, learning, and problem-solving (Nilsson, 1998). According to Baker and Smith (2019), AI is a broad notion that encompasses a wide range of technologies and algorithms. Earlier in its growth, $\mathrm{AI}$ in education was most typically connected with intelligent tutoring systems that tried to automatically address issues such as boosting operator efficiency (Hwang, 2003). The growth of computing and information processing methods has expedited the development of artificial intelligence (AI), which attempts to allow computers to accomplish tasks by emulating intelligent human behaviors such as inference, analysis, and decision making (Duan, Edwards, \& Dwivedi, 2019; Topol, 2019). In recent decades, researchers who have studied AI have reported significant progress. Using techniques like classical machine learning or modern deep learning, a growing number of products may provide "intelligent services" by inferring or acting like humans. AI is now used in several fields, such as visual and voice recognition, decision-making, natural language processing, and language translation, and comes in various ways, including computer programs, software, embedded control systems in equipment and robots. Some medical systems can assist human experts in identifying possible diseases or making decisions by processing a vast range of data (Lathuiliere, Massé, Mesejo, \& Horaud, 2019; Okuno, Nakadai, \& Kitano, 2002; Jantakoon \& Wannapiroon, 2017), and some robots can interact with humans using visual and audio monitoring technologies (Lathuiliere, Massé, Mesejo, \& Horaud, 2019; Li, Li, \& Niu, 2020; Zhu, 2020). AI technologies have been used to advance a wide variety of businesses in today's society (Russell \& Norvig, 2018). In this analysis, we restrict the definition of Artificial Intelligence to the educational context. AIEd implementations are divided into three groups by Baker and Smith (2019): learner-oriented AIEd, instructor-oriented AIEd, and institutional system-oriented AIEd. Students may research a topic using a learner-oriented AI, often known as an adaptive or customized learning management system. An instructor-oriented tool can automate administrative processes, reviews, plagiarism detection, and feedback provision. These systems may also aid instructors in keeping track of their student's progress so that proactive intervention can be given. With improvements such as personalized ITS (Steenbergen-Hu \& Cooper, 2014), support systems are becoming more sophisticated (Heffernan \& Heffernan, 2014), and "Instructor bots," AIEd has 
demonstrated its ability to help learners recognize knowledge gaps and obtain personalized support, freeing instructors from everyday menial tasks and allowing them to respond to students more effectively (Bayne, 2015). Finally, AIEd resources may provide valuable information to institutional managers and decision-makers, such as enrolment and retention rates across disciplines or universities. AIEd's application is still a hot topic among academics. Artificial intelligence in education (AIED) has opened new avenues for developing more successful learning practices and improved technology-enhanced learning applications or environments (Jantakun \& Jantakoon, 2021; Jantakoon et al., 2019). Most scholars and professionals in the fields of computers and education, on the other hand, continue to struggle to implement related practices or programs (Kay, 2012). Intelligent tutoring systems and advanced learning systems face difficulties in terms of computer programming abilities and strategies for simulating human experts' wisdom. Human tutors' knowledge and understanding were used to make judgments and assessments based on the best possible data to help overcome challenges and enhance learning for particular students. The strong dependency on technologies and the cross-disciplinary structure of AIED creates these difficulties. If researchers do not grasp the functions of AI in education and how AI programs function, they will not successfully incorporate AIED applications and practices and raise and analyze critical AIED research concerns. An AI application may, for example, act as a mentor, monitoring student learning processes, assessing results, and offering on-demand assistance. Interdisciplinary teams can develop intelligent teaching programs focused on their students' needs, helping students to study, practice, and interact with peers or tutors while also offering guidance, advice, and help to those who follow. Their situation or requirements. The know-how and features of AI technology, on the other hand, allow university instructors to use effective AI applications in the classroom to increase learning outcomes, inspiration, or student participation as researchers. The effect of AI applications may be studied in education. The architecture of AI algorithms with humanity as the principal concern about the state of human beings is AI; this kind of AI involves explanatory and interpretable processes for computation and decision and continuous modifications of AI algorithms through human contexts and society to increase the mind of human beings through machines, thus increasing human well-being.

In these sections, we propose a standard paradigm for higher education artificial intelligence and the concept and features of AI technology. Test issues are discussed in a common framework for artificial intelligence in higher education, which will lead researchers and educators to develop and implement Best Practice as an educational management method to allow educators or managers to prepare, make decisions and adapt the model implementations in the future.

\section{Objective}

1) To develop a common framework for artificial intelligence in higher education (AAI-HE model).

2) To evaluate the AAI-HE model.

\section{Scope of the Study}

\section{1) Population}

The population is the experts in the field of artificial intelligence in higher education

2) Samples Groups:

Samples are five experts in the field of artificial intelligence in higher education chosen by purposive sampling. They are highly experienced experts in these fields for at least five years.

3) Variables of the study:

1) Independent variable is the AAI-HE model.

2) Dependent variable is the appropriateness of the AAI-HE model.

\section{Method}

\subsection{Phase 1 develop an AAI-HE model}

Develop an AAI-HE model, components and technology of AI, roles for artificial intelligence in education, machine learning, and deep learning, DSS modules, and AI to enhance campus efficiencies.

\subsection{Phase 2 Assessment}

1) Develop the AAI-HE model.

2) Assessment of the appropriateness of the AAI-HE model. The output data was obtained by using a 5-point Likert Scale-based suitability measurement scale as well as means $(\bar{x})$ statistics.

1.00-1.49 means the assessment topic is absolutely inappropriate 
1.50-2.49 means the assessment topic is inappropriate

2.50-3.49 means the assessment topic is neutral

3.50-4.49 means the assessment topic is appropriate

4.50-5.00 means the assessment topic is absolutely appropriate

\section{Results}

Stage 1. The AAI-HE model is composed of seven key components. Figure 1 depicts visual representations of these concepts

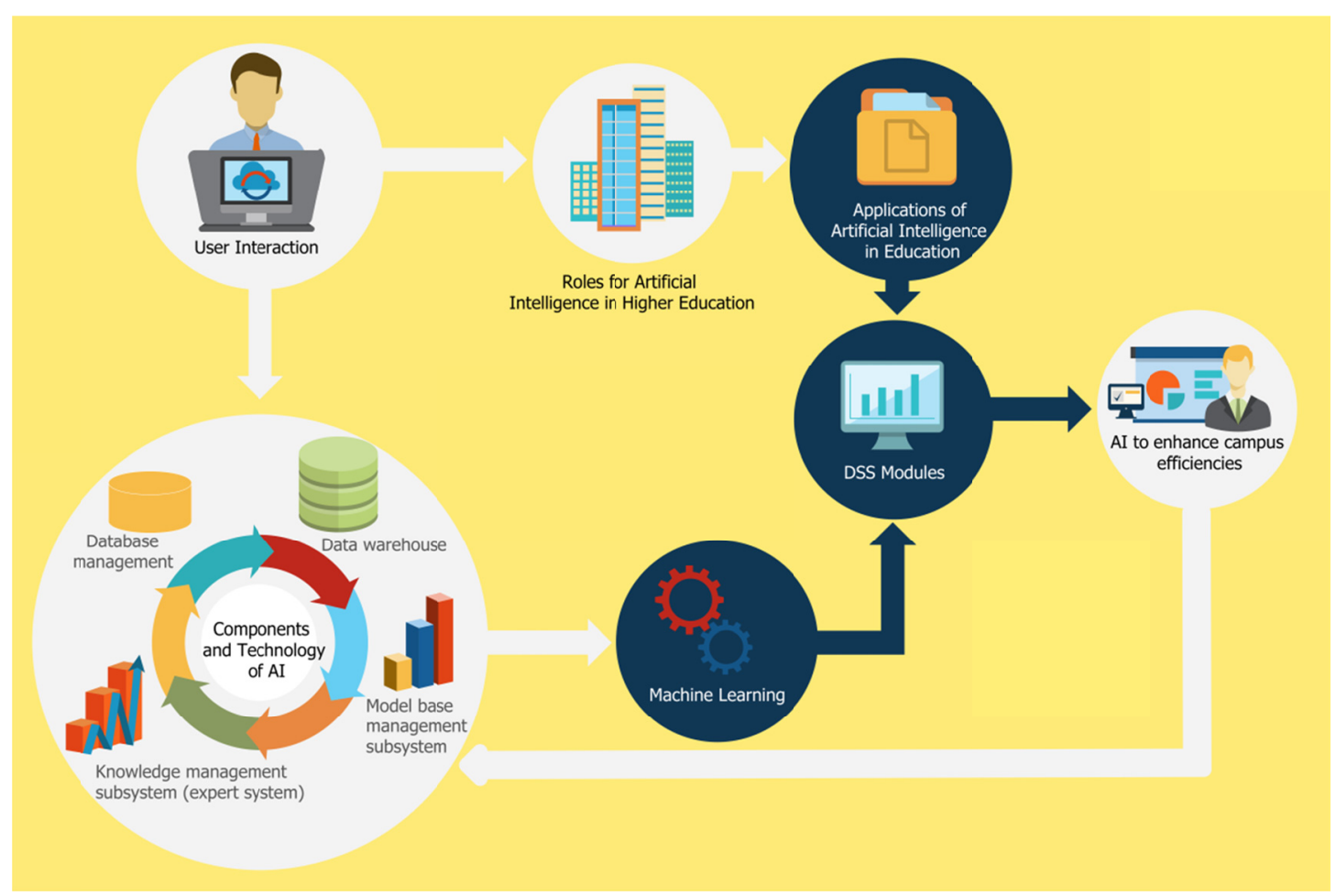

Figure 1. AAI-HE model

\subsection{User Interactive Components and Technology of AI}

Concerning information systems that include sophisticated algorithms, such as artificial intelligence, there are additional or fewer issues to deal with than systems with simpler ones. Reinventing assessment and methodologies could be essential when developing AI-driven websites. The result of these hurdles shouldn't make us condemn AI; instead, it should drive the advancement of Human Computer Interactions. Progression on these topics would be beneficial for non-iterative approaches.

\subsection{Components and Technology of AI}

\subsubsection{Data Management Subsystem}

1) Database: Although the database gathers valuable information to satisfy the demands of the company and enables more people to access it, they are also able to use it. Concepts and proposals can be general or raw data; texts, photographs, maps, and sound can be made up of concrete or abstractions. Data can be separated into three classes: internal data, off-site data, and personal data. Storage approach: The data structure of a database can be loosely categorized as relational, hierarchical, and network. That the majority of management information is stored in relational databases such as Oracle and Microsoft SQL, as with NoSQL, such as MongoDB and CSV.

2) Database management system: Database management manages, constructs, and modifies, and uses databases. A good database and its efficient administration can facilitate a multitude of managerial practices. Predictive modeling often involves typical three actions, such as browsing documents, establishing, and managing a complex collection of relationships, as well as producing reports. 
3) Data catalog: Data catalogs are a complete listing of the information in a database. Where the database stores the databases, including table and data files, is stored, is often called the database storage, and is part of the database definition. The key role is to provide explanations regarding the sources of data. Additionally, it encourages the addition, removal, and recovery of artifacts that have unique likes and dislikes.

4) Query function: Query does much of the work: retrieving, processing, and obtaining results. Other DSS components decide how data is obtained, directly articulate how the outcomes are reached, and pass them on those specifics to the person who made the request

\subsubsection{Data Warehouse}

1) Data Source: Data sources are usually coming from different distribution systems, including the operating and web transaction databases as well as external sources, making them sources for the data warehouse.

2) Extraction, transformation, and load tools: The goal of data collect data from the source, arrange it, validate it, and process it in the data warehouse, load it from the database.

3) Data modeling tool: It builds a knowledge model for the source data warehouse, and maintains an accurate depiction of the data in the aim.

4) Central repository and data mart: This contains the information needed to store and manage data models, as well as metadata. The metadata specifies the information in the source data warehouse, which can be used to re-transform the source data, and helps identify the mechanism by which it will be transposed to the target. In certain larger implementations, dedicated multidimensional databases can provide some advantages for multi-dimensional decision support. DSS programs should be part of a data mart. However, the features of the data center have been maintained even though the data in the data mart is not.

5) Target database: The goal of the target database is to contain, test, process, and order information.

6) Front-end data access and analysis tools: Information on the front-end user interface and behind the scenes provide market leaders and thinkers with access to data for more in-depth research.

7) Data warehouse management tools: Information processing includes computer protection, data security, and other issues as well

\subsubsection{Model Management Subsystem}

1) Model base: General, quantitative, management, other quantitative, and predictive models are all in the library. These mathematical models have DSS. The model has four bases that can induce, act, and operate on, construe, design, and critique models. Strategic, practical, and theoretical as well as models, there are also infrastructure projects and programming objects.

2) Modeling tool: In the event of semi-structured or unstructured problems, templates, such as Java, OLAP applications, or spreadsheets, certain model-specific tools such as programming models or spreadsheet modeling are also needed.

3) Model base management system: Using programming languages, DSS tools, and other low-level building blocks, are used to develop new programs and to improve them, as well as to manipulate the data

4) Model catalog: Also, the catalog can serve the same purpose as the reference model, which is to classify all models. Defining a model requires the model's key purpose is to see how well the design concept will function in the real world.

5) Execution, composition, and instructions: Since models have processes as well, they still have real-world limitations. It incorporates models that of that you may want to integrate as needed (for example, managing the output of a model; For example, predictions are processed by another model, such as the linear programming model).

\subsubsection{Knowledge Management Subsystem}

1) Knowledge acquisition: The collection, transmission, and then conversion and translation of information from experts or archives of knowledge to computers to new systems or databases are crucial functions of work on the creative continuum. Potential intelligence sources of information include researchers, textbooks, video archives (whether public or private), and databases. The difficult part of getting information is for the experts to agree on a price. We can't create a huge infrastructure without someone who communicates with human experts. However, in the majority of information engineering solutions, responses to human issues are interpretable, analogies for other problems are created, and challenges are constructed to provide counterexamples to explain the differences. 
2) Knowledge base: Our database has the information necessary to decipher, demonstrate, and resolve issues. It has two basic parts: skill and exploration. An in-depth fact is a fact in the solution of a certain domain, whereas a specific heuristic or guiding principle is a unique solution in a particular area of application.

3) Inference engine: In expert systems, the "brains" are regulated structures, also known as rule-makers, that have their working models, which can be called "inference engines." it is, in essence, a reasoning and diagramming tool that uses information from a knowledge base and a blackboard framework

4) User interface: A machine with an expert system can help to break down problems into a simplified form that people can better understand, allowing them to communicate with it more effectively. This can be completed using ordinary language. Since modern communication technologies cannot adapt to meet all of the requirements, most applications rely on a consumer to ask questions to find out what he or she wants. Additional time-sensitive tools such as spreadsheets, brochures, menus, and graphs may be used to increase productivity.

5) Blackboard: Blackboard architecture is used in work-memory computing. The checklist is used to provide a representation of the current issue through the use of data. It is used to make calculations between expectations and decisions. To-free (actual action) (candidate assumptions and action processes generated by the system so far).

6) Explanation subsystem: The ability to trace conclusions back to their origins is essential to making deductions and discovering problems. Using interactive questioning, the interpretation subsystem can help users make sense of their decisions and plans.

7) Knowledge-refining system: Human authorities have a methodology, that is, they can critically evaluate their human methodology and progress with the findings, In the same manner, analyses of the effectiveness or failure of programs are essential in the field of computerized learning so that they can better understand the reasons for it. Having a more precise knowledge base and improved logic improves knowledge recall and recall performance.

\subsection{Roles for Artificial Intelligence in Education}

\subsubsection{Artificial Intelligence Can Automate Basic Activities in Education}

Teaching undergraduates is difficult and time-consuming, regardless of how much or what or how many TAs grade assignments and exams for big lecture classes. While they devote considerable time to marking, you still spend a large amount of time with your students, especially in the lower grades, teaching, as well as time on professional development.

Although true that AIs will never be able to substitute for human grading, it is rapidly approaching that level. Using applications to grade with almost all sorts of multiple-choice and fill-in-in-the-the-the-blank training is just the beginning. Currently, essay grading software is in its early stages, but it will develop in the future and give Instructors more time to spend on class activities.

\subsubsection{Educational Software Can Be Adapted to Student Needs}

Artificial intelligence will radically impact education in the form of more individualized instruction on some of this. We've also seen adaptive learning and videogames serving as adjuncts. These programs adapt to the changing student needs, incorporating a greater focus on specific subjects, providing extensive practice for previously unnumbered subjects, and empowering students to follow their progress however they choose.

Instruction-mode customized to meet the needs of students of varying capabilities may be a machine-assisted method for making all levels grow together in one classroom some uses of digital technology have already had a big effect on education, and as AI develops in the future, adaptive programs can only be useful and common.

\subsubsection{It Can Point Out Places Where Courses Need to Improve}

Often instructors don't know what they don't know and thus fail to highlight or point out issues with their students may not see in their lessons. Since the solutions already exist, creative and innovative uses of artificial intelligence are also generating ideas Coursera is using big MOOCs. When students are all discovered to have given incorrect answers on a homework assignment, the machine notifies the teacher with a solution tailored message so they won't duplicate their errors.

\subsubsection{Students Could Get Additional Support from AI Tutors}

Though there will inevitably be more stuff that teachers can't deliver yet, the future will see more students educated by "tutor-machines" (the non-physical) Artificial intelligence-based tutorials and tutorials have since been created to assist students in elementary arithmetic, literature, and other subjects.

Programs will give students foundational skills, but real-world instructors must complement them with higher-order thought and imagination. Only because these problems cannot be solved in a short time does not mean 
they cannot be solved in the future. From recent technical progress, the use of state-of-of-the-the-the-art tutoring services is now beyond the realm of possibility.

\subsubsection{AI-Driven Programs Can Give Students and Educators Helpful Feedback}

Students are as well as professors have more control of their coursework which has the added benefit of AI benefits. Improvement in the assessment of academic progress also includes reviews on the program's overall effectiveness. AI is used in both on- and off-campus classrooms to measure students' success and inform teachers about any issues they might be having. This kind of AI assists students in providing the kind of service they need. educators view possible problems with instruction for students who might be experiencing difficulties with learning Since the AI systems focus on providing individual course guidance, they're about all aspects of life. Some academics are designing programs that allow students to determine which majors have been fruitful and underdeveloped areas of study. Future students will no longer be trained but will need to be prepared for a bold new landscape of college majors.

\subsubsection{It Is Altering How We Find and Interact With Information}

Knowledge smarts like this make it possible for us to work on both personal and career matters, as well as how we go about our educational pursuits. The use of artificial intelligence would alter the way knowledge is stored and accessed in future students. Students today are perfectly willing to do something as long as it's "creative".

\subsubsection{It Could Change the Role of Instructors}

AI instructors will step in when necessary to give students the freedom to explore on their while also providing plenty of instructional support and using their insights to the class. Technology is a significant contributor to many of these changes in the classroom, particularly in the rapidly growing segment of schools that have fully embraced the flipped learning method. Since intelligent systems are now commonplace in education, there will still be an ongoing debate about the appropriate nature of what Instructors should be doing and how they should be evaluated, so it will continue to be a topic for leaders and organizations for some time. We have already mentioned that artificial intelligence will take on such activities as grading, including giving the pupil a better learning experience, and also serve as a replacement for real-world tutoring. Though AI may be used in many other areas, it should not be seen as a panacea for all problems in education. Artificial intelligence devices may be designed to be an instrument for students to help find answers and do fundamental research for instructors. If in most cases artificial intelligence (AI) shifts the facilitator's position, instructors would be dispensable.

\subsubsection{AI Can Make Trial-And-Error Learning Less Intimidating}

A lot of trial and error is involved in all forms of study. Failure or not understanding the solution is a huge obstacle for many students. Some people get upset when they are placed in positions of prominence, such as when they are required to meet influential individuals like teachers or bosses. Placing sophisticated software to assist students in learning is much more appealing than coping with the pain of trial and error. AI offers students an experiment that can be conducted in a free and relaxed way, particularly when it comes to learning. Trial and error is, of course, the natural process for AI.

\subsubsection{Data Powered by AI Can Change How to Support Students}

Artificial intelligence has changed the way colleges are gathering and delivering data on their prospective and existing students. At any level, computer, from attracting students to guiding them across the curriculum, the computer science department makes the college more customizable to the needs and interests of students.

\subsubsection{AI May Change Where Students Learn, Who Teaches Them, and How They Acquire Basic Skills}

This initiative will put in place a digital platform that would allow students to learn from anywhere in the world, using software and help from mentor support to replace human teachers at times when they aren't in class. Digital teaching systems improve fundamental abilities. When the popularity of these programs and the individuals with whom they provide a close relationship grows, moreover, the variety of resources they have tends to broaden.

\subsection{Machine Learning and Deep Learning}

\subsubsection{Machine Learning}

The goal of machine learning is to produce predictions that are very strong, insightful, and reliable out of unsupervised and complex data. Statistical theory is closely intertwined with the best current thinking in machine learning. While also model inference is conducted using models, however, statisticians rely on interpreting their models' parameters. The ML community's major preoccupation was to get the best possible result, regardless of consequences. Both measurement tools must be optimized to guarantee the accuracy of the experiment. An "out of 
sample" that predicts new data. To keep machine learning more accessible, there is also a new movement to make model structures clearer. These models have been built according to the same standard as in the past and will work in the future.

\subsubsection{Deep Learning}

The models of Deep Learning use a deep learning transformation: they do one input-specific job at a time, one at a time. When you're making a video, you use CNN. If you want to take advantage of text data, you must apply the text to the context in the room. This can be done by word2vec transformations (short-long term memory sequences) or LSTM for each new hidden Markov model that is created and the LTM-like mechanisms built daily, an infinite number of new architectures can also be developed every day.

\subsection{DSS Modules}

1) Students Module: Enrollment of students, Reclassification of studies, tuition, specialization selection Scholarships, dorms for students Certificates are given, Data and announcements on the web, The transfer of students, Students are being expelled. Interrupting research, extending studies, doing a second or more specialty, in other institutions, there is a diploma test. Activities such as tutorials and career counseling.

2) Teaching Module: Materials, syllabi, and instruction Schedule of classes choosing elective classes tutoring activities for teaching and studying the practical job of students, Bachelor's degree preparation, exams, etc. The teaching team is being evaluated. Exam timetable, Final examinations, as well as partial tests during the semester managing the grading of students, Bachelor final exam, and contestation of assessment findings.

3) Research Module: Evaluation of scientific studies, efficiency problems, and expectations coefficients of pay, Human capital management approach, Job postings and interviews for research jobs, Ph.D. operation and assessment, grant administration and continuity, and so on.

\subsection{Applications of Artificial Intelligence in Education}

\subsubsection{Personalized Learning}

Artificial intelligence is being used to personalize learning for each pupil. Making use of the principle of Hyper-Personalization AI technologies, enabled by machine learning, are combined to create a personalized learning profile for each student and to tailor training materials based on the preferred learning mode. Student skills and knowledge vary. To help students understand a lecture, instructors can break it into mini learning guides, smart notes, or flashcards.

With AI assisting in the development of digital material, learning is becoming more digitized and less dependent on paperbacks. There will therefore be fewer written materials. AI-powered applications and systems allow students to get quick and personalized answers, as well as clear tutor doubts. AI is also used to improve tutoring and the design of assistants. Personal learning conversations that may help with studies or assignments Smart instructional programs, such as Carnegie Learning, offer immediate guidance and interact closely with students. These research assistants are still working to develop the integrated learning function so that all students can learn at their level and at the appropriate time.

\subsubsection{Voice Assistants}

Voice assistants, such as Amazon's Alexa, Apple Siri, Microsoft Cortana, and others, are another AI feature that educators can use successfully in learning. Students can converse with media using these voice assistants. Learn without the assistance of a teacher. They will work at home and outside of school to foster encounters with learning materials or to gain access to external learning assistance. In the case of higher education, conventional learning approaches are gradually being phased out, with many universities and colleges providing voice assistants to their students instead of traditional handwritten student handbooks. Or a complicated website to help with university-related knowledge needs.

\subsubsection{Aiding Educators in Administrative Tasks}

Educators deal for more than just their jobs in an educational environment. However, it also entails maintaining the classroom atmosphere and performing a broad variety of corporate activities. They are tasked with a variety of non-teaching responsibilities, such as essay evaluation, paper marking, test handling, handling necessary papers, overseeing HR and staffing matters, and staging and handling classroom supplies. Booking and field management responsibilities are managed. Travel, parent engagement and reaction, interaction assistance and second language issues, patient or absenteeism monitoring, and provision of a learning environment. 


\subsubsection{Breaking Barriers}

Artificial intelligence technologies and devices have aided in making global schools accessible to everyone, regardless of language or disability. There are all-inclusive services.

\subsection{AI to Enhance Campus Efficiencies}

\subsubsection{Colleges Look to AI to Power Predictive Analytics}

AI holds promise for universities as a way to drive student outcomes and empower personalized learning. Although there has been much discussion about the opportunity here, real adoption has yet to reach the mainstream: Rather, higher education is poised to take the lead.

\subsubsection{Blended Data Centers Continue to Support Campuswide Computing}

As part of the broader cloud transformation, many institutions continue to use a hybrid approach to data center operations, mixing public cloud services with on-premises technologies.

\subsubsection{Phishing and Ransomware Remain Key Campus Threats}

All of this makes higher education an enticing target, and with identities stolen as a result of phishing attacks, ransomware is becoming a more plausible attack scenario.

\subsubsection{Colleges Look to CRM Solutions for Student Lifecycle Management}

This software applications promise to provide administrators with a comprehensive vision of the student environment to help them achieve their goals.

\subsubsection{Smart Campus Technologies Offer Cost-Savings Potential}

Higher education is embracing the intelligent sector, a vision of a technologically integrated learning area that combined data and devices with the development of smart municipalities, to increase student experience. Colleges ought to be smarter to succeed. A data-driven campus could allow managers to save money by simplifying costs. The quick and intuitive interaction between university structures and management due to networking can be found by students and faculty. Data could help improve public safety through monitoring and warning systems. Improved usability could help students learn more efficiently in the classroom.

Stage 2. The evaluation of the AAI-HE model's results.

Table 1. The assessment of systems principles, concept, and objective

\begin{tabular}{lccc}
\hline \multirow{2}{*}{ Evaluation Lists } & \multirow{2}{*}{ Level of suitability } & \multicolumn{2}{c}{ Level of assessment } \\
\cline { 3 - 4 } & & $\overline{\mathrm{x}}$ & S.D. \\
\hline 1. System's principle and concept & absolutely appropriate & 4.60 & 0.54 \\
2. System's objective & absolutely appropriate & 4.80 & 0.44 \\
Overall & absolutely appropriate & 4.70 & 0.48 \\
\hline
\end{tabular}

The following tables describe the assessment of systems principles, concept, and objective. The assessment of systems principle, concept, and objective from the experts is absolutely appropriate $(\overline{\mathrm{x}}=4.70$, S.D. $=0.48)$.

Table 2. The module assessment

\begin{tabular}{lccc}
\hline \multirow{2}{*}{ Evaluation Lists } & Level of suitability & \multicolumn{2}{c}{ Level of assessment } \\
\cline { 3 - 4 } & & $\overline{\mathrm{x}}$ & S.D. \\
\hline 1. User Interactive Components and Technology of AI & appropriate & 4.40 & 0.54 \\
2. Components and Technology of AI & absolutely appropriate & 4.80 & 0.44 \\
3. Roles for Artificial Intelligence in Education & absolutely appropriate & 4.60 & 0.54 \\
4. Machine Learning and Deep Learning & absolutely appropriate & 4.80 & 0.44 \\
5. DSS Modules & absolutely appropriate & 4.60 & 0.54 \\
6. Applications of Artificial Intelligence in Education & appropriate & 4.40 & 0.54 \\
7. AI to enhance campus efficiencies & absolutely appropriate & 4.80 & 0.44 \\
Overall & absolutely appropriate & 4.63 & 0.49 \\
\hline
\end{tabular}


The results are presented in Table 2. The module assessment by the experts is absolutely appropriate $(\overline{\mathrm{x}}=4.63$, S.D. $=0.49)$.

Table 3. The assessment of AI to enhance campus efficiencies

\begin{tabular}{llcc}
\hline \multirow{2}{*}{ Evaluation Lists } & \multirow{2}{*}{ Level of suitability } & \multicolumn{2}{c}{ Level of assessment } \\
\cline { 3 - 4 } & & $\overline{\mathrm{x}}$ & S.D. \\
\hline 1. Colleges Look to AI to Power Predictive Analytics & absolutely appropriate & 4.80 & 0.44 \\
2. Blended Data Centers Continue to Support Campus wide Computing & absolutely appropriate & 4.80 & 0.44 \\
3. Phishing and Ransomware Remain Key Campus Threats & appropriate & 4.40 & 0.54 \\
4. Colleges Look to CRM Solutions for Student Lifecycle Management & absolutely appropriate & 4.60 & 0.54 \\
5. Smart Campus Technologies Offer Cost-Savings Potential & absolutely appropriate & 4.80 & 0.44 \\
Overall & absolutely appropriate & 4.68 & 0.47 \\
\hline
\end{tabular}

The assessment of AI to enhance campus efficiencies from the experts is absolutely appropriate $(\overline{\mathrm{x}}=4.68, \mathrm{~S} . \mathrm{D} .=$ 0.47).

Table 4. Summarizes the results of Table 3 details the values used in the assessment of AI to enhance campus efficiencies

\begin{tabular}{lrrr}
\hline & & \multicolumn{2}{c}{ Level of assessment } \\
\cline { 3 - 4 } Evaluation Lists & Level of suitability & $\overline{\mathrm{x}}$ & S.D. \\
\hline 1. The assessment of systems principle, concept, and objective & absolutely appropriate & 4.70 & 0.48 \\
2. The module assessment & absolutely appropriate & 4.63 & 0.49 \\
3. The assessment of AI to enhance campus efficiencies & absolutely appropriate & 4.68 & 0.47 \\
Overall & absolutely appropriate & 4.67 & 0.48 \\
\hline
\end{tabular}

The results are presented in Table 4 . The summary of the results is absolutely appropriate $(\overline{\mathrm{x}}=4.67$, S.D. $=0.48)$.

\section{Conclusion and Discussion}

The composition of the AAI-HE model derived from the design of seven groups of service providers can be described as follows: 1) User Interactive Components and Technology of AI 2) Components and Technology of AI consists of the following Data Management Subsystem, Data Warehouse, Model Management Subsystem, and Knowledge Management Subsystem 3) Roles for Artificial Intelligence in Education 4) Machine Learning and Deep Learning 5) DSS Modules consist of the following Students Module, Teaching Module, and Research Module 6) Applications of Artificial Intelligence in Education consists of the following Personalized Learning, Voice assistants, and Aiding educators in administrative tasks 7) AI to enhance campus efficiencies consists of the following Colleges Look to AI to Power Predictive Analytics, Blended Data Centers Continue to Support Campuswide Computing, Phishing and Ransomware Remain Key Campus Threats, Colleges Look to CRM Solutions for Student Lifecycle Management, and Smart Campus Technologies They Offer Cost-Savings Potential. The assessment result of the suitability of the composition of the AAI-HE model from five experts reveals that the suitability average level is absolutely appropriate. This demonstrated that the system's composition derived from the design could be improved as the AAI-HE model to guide researchers and instructors who intend to study, implement, Best practices as a tool to support the management process so that administrators could set up plans and make any decisions more efficiently. This is consistent with Klutka et al. (2018), who found that the implementation of artificial intelligence had the most significant ability to transform higher education of any technological advancement. Furthermore, the findings are consistent with those of Kaku (2011) and Kelly (2017), who discovered that the advancement of artificial intelligence, deep learning, and computational architectures, and some continue to predict a brighter future for AI across all sectors of society. In terms of fundamental technologies, network systems, and essential equipment needed for internet connectivity, the AAI-HE Model should be well equipped.

\section{References}

Baker, T., \& Smith, L. (2019). Educ-AI-tion rebooted? Exploring the future of artificial intelligence in schools and colleges. Retrieved from https://media.nesta.org.uk/documents/Future_of_AI_and_education_v5_WEB. pdf 
Bayne, S. (2015). Teacherbot: interventions in automated teaching. Teach. High. Educ., 20(4), 455-467. https://doi.org/10.1080/13562517.2015.1020783

Duan, Y., Edwards, J. S., \& Dwivedi, Y. K. (2019). Artificial intelligence for decision making in the era of Big Data-evolution, challenges, and research agenda. International Journal of Information Management, 48, 63-71. https://doi.org/10.1016/j.ijinfomgt.2019.01.021

Heffernan, N. T., \& Heffernan, C. L. (2014). The ASSISTments ecosystem: Building a platform that brings scientists and teachers together for minimally invasive research on human learning and teaching. Int. J. Artif. Intell. Educ., 24(4), 470-497. https://doi.org/10.1007/s40593-014-0024-x

Jantakoon, T., \& Wannapiroon, P. (2017). System architecture of business intelligence to aun-qa framework for higher education institution. Turkish Online Journal of Educational Technology, 2017(November Special Issue INTE), 1045-1052.

Jantakoon, T., Wannapiroon, P., \& Nilsook, P. (2019). Virtual Immersive Learning Environments (VILEs) Based on Digital Storytelling to Enhance Deeper Learning for Undergraduate Students. Higher Education Studies, 9(1), 144-150. https://doi.org/10.5539/hes.v9n1p144

Jantakun, T., \& Jantakoon, T. (2021). Digital Educational Computer Games Environments Supporting Education (DECGE-SE). Higher Education Studies, 11(2), 91-98. https://doi.org/10.5539/hes.v11n2p91

Kaku, M. (2011). Physics of the future: The inventions that will transform our lives. London: Penguin Group.

Kay, J. (2012). AI and education: Grand challenges. IEEE Intelligent Systems, 27(5), 66-69. https://doi.org/10.1109/MIS.2012.92

Kelly, K. (2017). The inevitable: Understanding the 12 technological forces that will shape our future. London: Penguin Group

Klutka, J., Ackerly, N., \& Magda, A. J. (2018). Artificial intelligence in higher education: Current uses and future applications. Wiley education services.

Lathuiliere, S., Masse, B., Mesejo, P., \& Horaud, R. (2019). Neural network-based reinforcement learning for audio-visual gaze control in human-robot interaction. Pattern. https://doi.org/10.1016/j.patrec.2018.05.023

Li, J., Li, P., \& Niu, W. (2020). Artificial intelligence applications in upper gastrointestinal cancers. The Lancet Oncology, 21(1), 1-4. https://doi.org/10.1016/S1470-2045(19)30721-1

Nilsson, N. J. (1998). Artificial Intelligence: A New Synthesis. San Francisco: Morgan Kaufmann Publishers, Inc.

Okuno, H. G., Nakadai, K., \& Kitano, H. (2002). Social interaction of humanoid robot based on audio-visual tracking. In International conference on industrial, engineering and other applications of applied intelligent systems (pp. 725-735). Berlin, Heidelberg: Springer. https://doi.org/10.1007/3-540-48035-8_70

Russell, S., \& Norvig, P. (2018). Artificial Intelligence: A Modern Approach (global edition). Harlow, United Kingdom: Pearson Education Limited.

Steenbergen-Hu, S., \& Cooper, H. (2014). A meta-analysis of the effectiveness of intelligent tutoring systems on college student's academic learning. J. Educ. Psychol., 106(2), 331-347. https://doi.org/10.1037/a0034752

Topol, E. J. (2019). High-performance medicine: The convergence of human and artificial intelligence. Nature Medicine, 25(1), 44-56. https://doi.org/10.1038/s41591-018-0300-7

Zhu, H. (2020). Big data and artificial intelligence modeling for drug discovery. Annual Review of Pharmacology and Toxicology, 60, 573-589. https://doi.org/10.1146/annurev-pharmtox-010919-023324

\section{Copyrights}

Copyright for this article is retained by the author(s), with first publication rights granted to the journal.

This is an open-access article distributed under the terms and conditions of the Creative Commons Attribution license (http://creativecommons.org/licenses/by/4.0/). 